\title{
Anticoagulant Treatment of Cancer Patients in the Real World - Hematologists/Oncologists vs. Angiologists/Phlebologists
}

\section{Axel Matzdorff ${ }^{1 *}$, Bettina Ledig ${ }^{2}$, Markus Stuecker ${ }^{3}$ and Hanno Riess ${ }^{4}$}

${ }^{1}$ Department of Internal Medicine II, Asklepios Clinic Uckermark Auguststr, Schwedt, Germany

${ }^{2}$ Scientific Affairs LEO Pharma GmbH, Neu-Isenburg, Germany

${ }^{3}$ Vein Center of the Departments of Dermatology and Vascular Surgery, Ruhr-University Bochum, Bochum, Germany

${ }^{4}$ Medical Clinic Haematology/Oncology, Charité Campus Charite Mitte Chariteplatz, Berlin, Germany

Keywords: Cancer-associated venous thromboembolism; Health services research; Hematologist; Oncologist; Angiologist; Phlebologist

Cancer patients have an exceptionally high risk of venous thromboembolism (VTE). This is due to surgeries, immobility, advanced age, prothrombotic medications, and the release of procoagulant factors by the tumor itself. At the same time cancer patients have a high bleeding risk. Unfortunately there is only a handful of high-quality, randomized studies on how to anticoagulate patients with cancer-associated VTE. Despite this dearth of information guidelines uniformly recommend low molecular weight heparin (LMWH) over vitamin $\mathrm{K}$ antagonists (VKAs) for treatment of established acute VTE both initially and longterm (first 3-6 months) (Table 1).

This is because LMWHs are more effective without increasing the risk of bleeding. Use of direct oral anticoagulants (DOACs) is currently not recommended during active cancer treatment.

It is uncertain how consistently clinicians adhere to these recommendations considering the inconvenience of daily s.c. LMWH injections compared to oral treatment. Several recent publications suggest that observance of guideline recommendations is relatively high with respect to the use of LMWH for acute VTE but suboptimal in long-term treatment [1-4]. After the acute phase many patients are switched to oral anticoagulants.

In 2014 we sent a questionnaire to hematologists/oncologists and other VTE-specialists in Germany to assess current practice of VTE management in cancer patients. We found that similar to their colleagues in other countries LMWH is the treatment of choice for acute VTE but for long-term secondary prophylaxis many switch to VKAs and DOACs. Among those who continue LMWH long-term, the dose is often reduced to $75 \%$ or $50 \%$ of the initial dose [5]. In a subsequent analysis we compared the responses of hematologists/oncologists and angiologists/phlebologists [6]:

- Cancer-associated VTE was considered a common issue both in hematology/oncology and angiology/phlebology daily practice.

Initial treatment of established venous thromboembolism (VTE): First 10 days of anticoagulation

1. Low-molecular-weight heparin (LMWH) is recommended.

2. Fondaparinux and unfractionated heparin can also be used.

Early maintenance (10 days to $\mathbf{3}$ months) and long-term (beyond $\mathbf{3}$ months)

1. LMWHs are preferred over vitamin $\mathrm{K}$ antagonists (VKAs).

2. LMWH should be used for a minimum of 3 months.

3. Direct oral anticoagulants (DOACs) can be considered for patients with stable cancer not receiving systemic anticancer therapy, and in cases where VKA is an acceptable, but not an available, treatment choice.

4. After 3-6 months, termination or continuation of anticoagulation (LMWH, VKA or DOAC) should be based on individual assessment.

Table 1: Current guideline recommendations for treatment of venous thromboembolism in patients with cancer.
- For both initial treatment of established VTE and for secondary prophylaxis throughout the next 3-6 months both specialties prefer LMWH but a considerable percentage also switches to VKAs and DOACs.

- If hematologists/oncologists give LMWH for 3-6 months then they reduce the dose earlier than angiologists/phlebologists and they prefer half-therapeutic doses.

- Beyond 6 months there is no difference with regards to using LMWH or oral anticoagulants. Fewer angiologists/ phlebologists responded, they would prescribe DOACs to cancer patients (any indication, not only VTE).

The reasons for this are not clear. Angiologists/phlebologists might be more familiar with VTE guideline recommendations, since VTE is more central to their medical specialty while in hematology/oncology practice this is only one issue among many others. It could also be that hematologists/oncologists are more concerned about the risk of bleeding because this is particularly high in cancer patients. Shorter anticoagulation and half-therapeutic dosing 'feels' safer.

The main problem is that we do not have good data on how to manage cancer-associated VTE. In particular, we need more studies in "difficult" patients e.g. cancer patients with intensive chemotherapy or with a high bleeding risk from gastrointestinal tumors or with brain metastases. Cancer is a major contributor to global disease burden, affecting millions of patients worldwide every year and many will develop VTE. We need more studies on these common and real-world issues so that a cancer-associated VTE does not feel "difficult" any more.

\section{References}

1. Kahn SR, Springmann V Schulman S, Martineau J, Stewart JA et al. (2012) Management and adherence to VTE treatment guidelines in a national prospective cohort study in the Canadian outpatient setting. The recovery study. Thromb Haemost 108: 493-498.

2. Kleinjan A, Hutten BA, Di Nisio M, Büller HR, Kamphuisen PW (2014) Anticoagulant treatment of cancer patients with pulmonary embolism in the real world. Actual use of low-molecular-weight heparin in cancer. Neth $\mathrm{J}$ Med $72: 467-472$

*Corresponding author: Axel Matzdorff, MD, PhD, Department of Internal Medicine II, Asklepios Clinic Uckermark Auguststr. 2316303 Schwedt/Od. Germany, Tel: (03332) 534620; E-mail: a.matzdorff@asklepios.com

Received October 04, 2017; Accepted October 06, 2017; Published October 13 2017

Citation: Matzdorff A, Ledig B, Stuecker M, Riess H (2017) Anticoagulant Treatment of Cancer Patients in the Real World - Hematologists/Oncologists vs. Angiologists/ Phlebologists. J Oncol Transl Res 3: 120. doi: 10.4172/2476-2261.1000120

Copyright: (c 2017 Matzdorff A, et al. This is an open-access article distributed under the terms of the Creative Commons Attribution License, which permits unrestricted use, distribution, and reproduction in any medium, provided the original author and source are credited. 
Citation: Matzdorff A, Ledig B, Stuecker M, Riess H (2017) Anticoagulant Treatment of Cancer Patients in the Real World - Hematologists/Oncologists vs. Angiologists/Phlebologists. J Oncol TransI Res 3: 120. doi: 10.4172/2476-2261.1000120

3. Kleinjan A, Aggarwal A, Van de Geer A, Faselis C, Büller HR (2013) A worldwide survey to assess the current approach to the treatment of patients with cancer and venous thromboembolism. Thromb Haemost 110: 959-965.

4. Sevestre MA, Belizna C, Durant C, Bosson JL, Vedrine L, et al. (2014) Carmen investigators for the groupe francophone thrombose et cancer (GFTC). Compliance with recommendations of clinical practice in the management of venous thromboembolism in cancer: The CARMEN study. J Mal Vasc 39: 161-168.
5. Matzdorff A, Ledig B, Stuecker M, Riess H (2016) Practice patterns for prophylaxis and treatment of venous thromboembolism in German cancer patients. Oncol Res Treat 39: 194-201.

6. Matzdorff A, Ledig B, Stuecker M, Riess H (2017) Prophylaxis and treatment of cancer-associated venous thromboembolism-comparison hematologist/ oncologists versus angiologist/phlebologists. Oncol Res Treat 40: 138-140. 